\title{
Spontaneous emission induced entanglement and steady entanglement in two atomic system
}

\author{
Huiyun $\mathrm{XU}^{1}$ and Guohui Yang ${ }^{2, *}$ \\ ${ }^{1}$ College of Physics and Electronics Engineering, Shanxi University, \\ Taiyuan 030006, P.R. China \\ 2 School of Physics and Information Engineering, Shanxi Normal University, \\ Linfen 041004, P.R. China
}

Received 15 Octorber 2015; Accepted 20 November 2015

Published Online 1 March 2016

\begin{abstract}
We report possibility of generating entanglement between two atoms in free space when their spatial separation is on the order of wavelength or less. We show that these two atoms get coupled by photon exchange due to spontaneous decay, which in turn generates the atomic entanglement. By introducing the incoherent pumping, one can obtain the steady entanglement, and the incoherent pumping can overcome the decay of the atoms. Moreover, one can obtain the larger value of steady entanglement via proper tuning the incoherent pumping.
\end{abstract}

PACS: 42.50.Ct, 42.50.Md, 03.65.Ud

Key words: Quantum Entanglement, Spontaneous Emission, Photon Exchange.

\section{Introduction}

As is well known, due to the understanding of entanglement creation and possible applications in quantum computing, quantum entanglement has attracted more attentions in the past few years. There have been many proposals for creating atomic entanglement [1-3], meanwhile some notable experimental demonstrations have also been performed. In practice, every quantum system is often constrained by unavoidable interaction with its environment, this may lead to the dissipation and destruction of entanglement $[4,5]$. As a consequence, the entanglement will be zero even though the system is initially in the maximal entanglement state. In fact, apart from its traditional role of destruction of entanglement, dissipation can also create entanglement in some system [6,7].

${ }^{*}$ Corresponding author. Email addresses: sxlfyghlxr@126.com (G. H. Yang) 


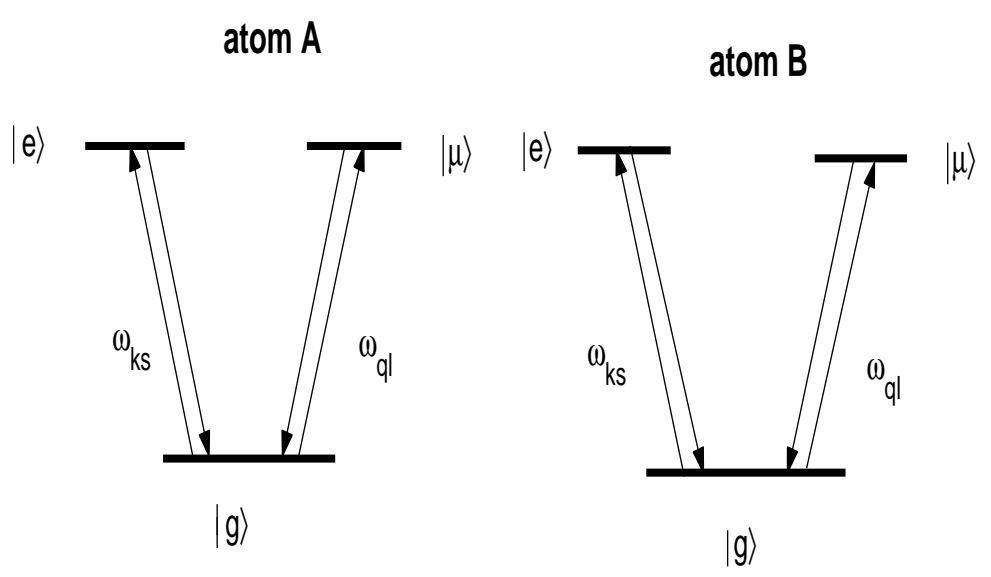

Figure 1: The atomic V-scheme and the two-atom configuration under consideration. The distance between the two atoms is considered to be small compared to the radiation wavelength.

Spontaneous emission in two-atom systems is an example of such noise which can destroy the entanglement [8]. However, beyond the conventional role of destroying entanglement, due to the possible photon exchange between atoms, the spontaneous emission also can create entanglement. In particular when the inter atomic distance is on the order of wavelength or less. It is known that a system of particles which are closer together than the relevant transition wave-length displays collective states which are immune against spontaneous emission. In this case there is a substantial probability that a photon emitted by one atom will be absorbed by the other and the photon exchange process can induce entanglement between atoms which partially overcome decoherence caused by spontaneous.

In our this paper, we extend this kind of studies to the case of pair of three-level atoms in $\mathrm{V}$ configuration. This system are coupled to a common thermostat at zero temperature and the reduced dynamics evolution is given by the semi-group $\left\{T_{t}\right\}$ of completely positive linear mappings acting on density matrices [9], this kind of dynamics takes into account only spontaneous emission and possibly photon exchange between atoms $[10,11]$.

\section{Theoretical treatment and results}

We consider two identical three-level atoms in $V$ configuration. The atoms (say $A$ and $B$ ) have two degenerate excited states $|e\rangle_{m},|\mu\rangle_{m}(m=A, B)$ and the ground state $|g\rangle_{m}$ as is shown in Fig. 1. The distance $R$ is small compared to the radiation wavelength. At such distances there is a substantial probability that the photons emitted by one atom will be absorbed by the other. As we known, for $V$ atomic configuration, usually the ndegenerate magnetic sublevels work as excited states. So, the dipoles in $|e\rangle_{m} \leftrightarrow|g\rangle_{m}$ and $|\mu\rangle_{m} \leftrightarrow|g\rangle_{m}$ are orthogonal to each other and also the polarization of the photon emitted 
from transition $|e\rangle_{m} \leftrightarrow|g\rangle_{m}$ is orthogonal to that emitted from $|\mu\rangle_{m} \leftrightarrow|g\rangle_{m}$ transition. Thus we will consider the photon emitted from $|e\rangle_{m} \leftrightarrow|g\rangle_{m}\left(|\mu\rangle_{m} \leftrightarrow|g\rangle_{m}\right)$ can only be absorbed by $|e\rangle_{m} \leftrightarrow|g\rangle_{m}\left(|\mu\rangle_{m} \leftrightarrow|g\rangle_{m}\right)$. Please note that an atom with V-scheme, having degenerate or nearly degenerate excited states, can also radiatively couple the orthogonal transitions when another identical atom is present within a distance of the radiation wavelength $[11,12]$. However, here we consider a different scheme where the two transitions $|e\rangle_{m} \leftrightarrow$ $|g\rangle_{m}$ and $|g\rangle_{m} \leftrightarrow|\mu\rangle_{m}$ are non-degenerate. So that we can ignore the cross coupling as mentioned above. Thus the dynamics of the system is given by the master equation

$$
\dot{\rho}=L_{I} \rho+L_{I I} \rho .
$$

Where the damping term $L_{I} \rho$ and $L_{I I} \rho$ can be expressed as following

$$
\begin{aligned}
L_{I} \rho= & \gamma_{1}\left(2 \sigma_{g e}^{A} \rho \sigma_{e g}^{A}-\sigma_{e e}^{A} \rho-\rho \sigma_{e e}^{A}\right) \\
& +\gamma_{2}\left(2 \sigma_{g \mu}^{A} \rho \sigma_{\mu g}^{A}-\sigma_{\mu \mu}^{A} \rho-\rho \sigma_{\mu \mu}^{A}\right) \\
& +\gamma_{1}\left(2 \sigma_{g e}^{B} \rho \sigma_{e g}^{B}-\sigma_{e e}^{B} \rho-\rho \sigma_{e e}^{B}\right) \\
& +\gamma_{2}\left(2 \sigma_{g \mu}^{B} \rho \sigma_{\mu g}^{B}-\sigma_{\mu \mu}^{B} \rho-\rho \sigma_{\mu \mu}^{B}\right), \\
L_{I I} \rho= & \Gamma_{1}\left(2 \sigma_{g e}^{B} \rho \sigma_{e g}^{A}-\sigma_{e g}^{A} \sigma_{g e}^{B} \rho-\rho \sigma_{e g}^{A} \sigma_{g e}^{B}\right) \\
& +\Gamma_{2}\left(2 \sigma_{g \mu}^{B} \rho \sigma_{\mu g}^{A}-\sigma_{\mu g}^{A} \sigma_{g \mu}^{B} \rho-\rho \sigma_{\mu g}^{A} \sigma_{g \mu}^{B}\right) \\
& +\Gamma_{1}\left(2 \sigma_{g e}^{A} \rho \sigma_{e g}^{B}-\sigma_{e g}^{B} \sigma_{g e}^{A} \rho-\rho \sigma_{e g}^{B} \sigma_{g e}^{A}\right) \\
& +\Gamma_{2}\left(2 \sigma_{g \mu}^{A} \rho \sigma_{\mu g}^{B}-\sigma_{\mu g}^{B} \sigma_{g \mu}^{A} \rho-\rho \sigma_{\mu g}^{B} \sigma_{g \mu}^{A}\right) .
\end{aligned}
$$

here $L_{I} \rho$ corresponds to the atom-vacuum field couplings leading to spontaneous, and $L_{I I} \rho$ represents the atom-atom coupling due to the possible photon exchange between atoms caused by spontaneous. Where $\sigma_{j g}^{m}=|j\rangle_{m}\langle g|(j=e, \mu)$ denote the atomic raising operators from $|g\rangle_{m}$ to $|e\rangle_{m}$ or $\left.\mu\right\rangle_{m}, \gamma_{1}\left(\gamma_{2}\right)$ is the spontaneous emission rate of transition $|e\rangle_{m} \rightarrow|g\rangle_{m}|\mu\rangle_{m} \rightarrow|g\rangle_{m}$, and $\Gamma_{1}$ and $\Gamma_{2}$ represent the photon exchange rate, they are the function of the distance $R$ between the atoms, under this case we know $\gamma_{1} \geq \Gamma_{1}$ and $\gamma_{2} \geq \Gamma_{2}$.

Now, let us study the entanglement property of this system caused by spontaneous emission. More precisely, we assume the atomic system are initially in the state $|e \mu\rangle$, here the two atom state $|i j\rangle \equiv|i\rangle_{A}|j\rangle_{B}(i, j=e, \mu, g)$, the corresponding density operator would be $\rho(0)=|e \mu\rangle\langle e \mu|$. For simplicity, the nine basis state corresponding to this atomic system are $|e e\rangle,|e \mu\rangle,|e g\rangle,|\mu e\rangle,|\mu \mu\rangle,|\mu g\rangle,|g e\rangle,|g \mu\rangle,|g g\rangle$. Thus the time evolution state $\rho=\left(\rho_{j k}\right)$ has the following form with respect to these basis

$$
\rho=\left(\begin{array}{ccccc}
\rho_{11(t)} & \cdots & \rho_{15(t)} & \cdots & \rho_{19(t)} \\
\vdots & & \vdots & & \vdots \\
\rho_{51(t)} & \cdots & \rho_{55(t)} & \cdots & \rho_{59(t)} \\
\vdots & & \vdots & & \vdots \\
\rho_{91(t)} & \cdots & \rho_{95(t)} & \cdots & \rho_{99(t)}
\end{array}\right) .
$$


Recalling the initial condition, and combined with the master equation (1), we could written out the differential equations of density matrix elements as following

$$
\begin{aligned}
\dot{\rho}_{22}= & -2\left(\gamma_{1}+\gamma_{2}\right) \rho_{22} \\
\dot{\rho}_{33}= & -2 \gamma_{1} \rho_{33}+2 \gamma_{2} \rho_{22}-\Gamma_{1}\left(\rho_{37}+\rho_{73}\right) \\
\dot{\rho}_{37}= & -2 \gamma_{1} \rho_{37}-\Gamma_{1}\left(\rho_{33}+\rho_{77}\right) \\
\dot{\rho}_{66}= & -2 \gamma_{2} \rho_{66}-\Gamma_{2}\left(\rho_{68}+\rho_{86}\right) \\
\dot{\rho}_{68}= & -2 \gamma_{2} \rho_{68}-\Gamma_{2}\left(\rho_{66}+\rho_{88}\right) \\
\dot{\rho}_{77}= & -2 \gamma_{1} \rho_{77}-\Gamma_{1}\left(\rho_{37}+\rho_{73}\right) \\
\dot{\rho}_{88}= & -2 \gamma_{2} \rho_{88}+2 \gamma_{1} \rho_{22}-\Gamma_{2}\left(\rho_{68}+\rho_{86}\right) \\
\dot{\rho}_{99}= & 2 \gamma_{1}\left(\rho_{33}+\rho_{77}\right)+2 \gamma_{2}\left(\rho_{66}+\rho_{88}\right) \\
& +2 \Gamma_{1}\left(\rho_{37}+\rho_{73}\right)+2 \Gamma_{2}\left(\rho_{68}+\rho_{86}\right),
\end{aligned}
$$

and the remaining matrix elements one can obtained by using the hermiticity condition of $\rho(t)$. We can also compute the explicitly value of these matrix elements by using the Laplace transform method, they are following

$$
\begin{aligned}
\rho_{22}(t)= & e^{-2\left(\gamma_{1}+\gamma_{2}\right) t} \\
\rho_{33}(t)= & \frac{e^{-2 \gamma_{1} t}}{2\left(\gamma_{2}^{2}-\Gamma_{1}^{2}\right)}\left[\gamma_{2}^{2} \cosh \left(2 \Gamma_{1} t\right)-\Gamma_{1} \gamma_{2} \sinh \left(2 \Gamma_{1} t\right)-\right. \\
& \left.2 \gamma_{2}^{2} e^{-2 \gamma_{2} t}+\Gamma_{1}^{2} e^{-2 \gamma_{2} t}+\gamma_{2}^{2}-\Gamma_{1}^{2}\right] \\
\rho_{37}(t)= & \rho_{73}(t)=\frac{\gamma_{2} e^{-2 \gamma_{1} t}}{2\left(\gamma_{2}^{2}-\Gamma_{1}^{2}\right)}\left[\Gamma_{1} \cosh \left(2 \Gamma_{1} t\right)-\gamma_{2} \sinh \left(2 \Gamma_{1} t\right)\right. \\
& \left.-\Gamma_{1} e^{-2 \gamma_{2} t}\right] \\
\rho_{77}(t)= & \frac{e^{-2 \gamma_{1} t}}{2\left(\gamma_{2}^{2}-\Gamma_{1}^{2}\right)}\left[\gamma_{2}^{2} \cosh \left(2 \Gamma_{1} t\right)-\Gamma_{1} \gamma_{2} \sinh \left(2 \Gamma_{1} t\right)\right. \\
& \left.-\Gamma_{1}^{2} e^{-2 \gamma_{2} t}+\Gamma_{1}^{2}-\gamma_{2}^{2}\right], \\
\rho_{88}(t)= & \rho_{33}(t)(1 \leftrightarrow 2), \quad \rho_{66}(t)=\rho_{77}(t)(1 \leftrightarrow 2), \\
\rho_{68}(t)= & \rho_{73}(t)(1 \leftrightarrow 2), \quad \rho_{86}(t)=\rho_{37}(t)(1 \leftrightarrow 2),
\end{aligned}
$$

where the explicit formula for the matrix element $\rho_{99}(t)$ is lengthy and tedious, we do not write it here. $1 \leftrightarrow 2$ means that subscript interchange.

Another interesting question is how to measure the amount of entanglement for a given quantum state. Since the damping causes the pure states evolve into mixed states, we need effective measure of mixed-state entanglement, one usually use the concurrence $[13,14]$, but in practice for a pair of $d$-level system here $d>2$, a computable measure of entanglement was proposed in Ref [15], which is defined as

$$
N(\rho)=\frac{\left\|\rho^{T_{A}}\right\|-1}{2}
$$




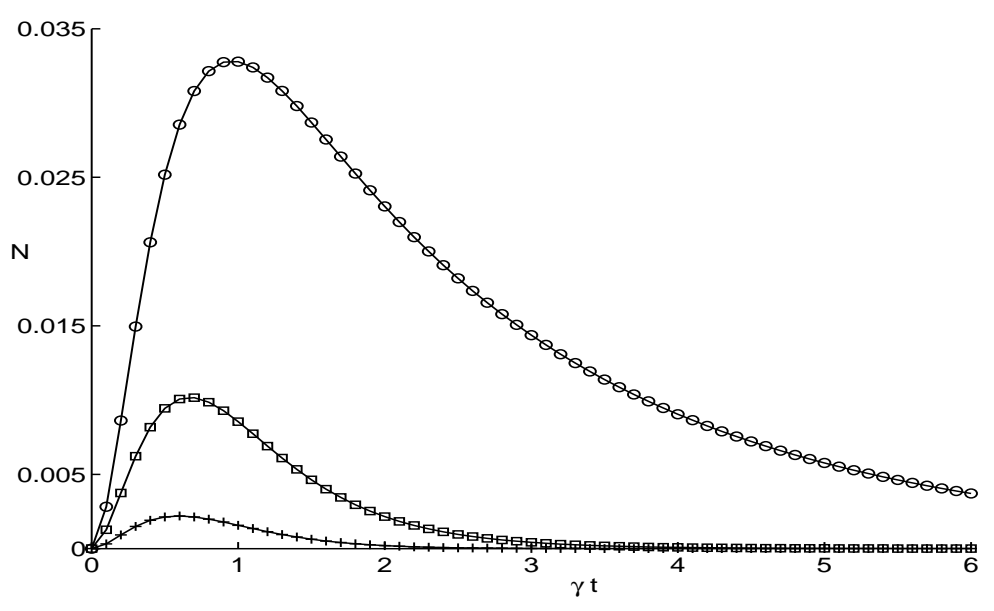

Figure 2: The negativity for our atomic system as a function of $t$ with different values of $\Gamma$. We set $\gamma_{1}=\gamma_{2}=\gamma=1$ and $\Gamma_{1}=\Gamma_{2}=\Gamma$. For the plus sign line: $\Gamma=0.3$, the square line: $\Gamma=0.6$, the circle line: $\Gamma=0.9$.

it is named negativity, and $\left\|\rho^{T_{A}}\right\|[16]$ is the trace norm of the partial transposition $\rho^{T_{A}}$ of the state $\rho$. The negativity is equivalent to the absolute value of the sum of the negative eigenvalues of $\rho^{T_{A}}$, i.e. $N(\rho)=-\sum_{i} \lambda_{i}$, where $\lambda_{i}$ are the negative eigenvalues of $\rho^{T_{A}}$. From the definition of the negativity, we note that it may be greater than one. But for different dimensions of density matrix the maximum value is different. To our system the maximum value of $N$ is 1 . After some calculation, we obtain the exact values of the eigenvalues of $\rho^{T_{A}}$ are

$$
\begin{aligned}
& \lambda_{1}=\lambda_{2}=0, \lambda_{3}=\rho_{22}(t), \lambda_{4}=\rho_{33}(t) \\
& \lambda_{5}=\rho_{66}(t), \lambda_{6}=\rho_{77}(t), \lambda_{7}=\rho_{88}(t) \\
& \lambda_{8,9}=\frac{\rho_{99}(t)}{2} \pm \frac{1}{2} \sqrt{\rho_{99}^{2}(t)+4\left[\rho_{37}(t)^{2}+\rho_{68}(t)^{2}\right]} .
\end{aligned}
$$

Now let us discuss the entanglement property of our atomic system, firstly from Eq. (8) we can check that only the eigenvalue $\lambda_{9}$ could be negative, and it is closely connect to the matrix element $\rho_{37}(t), \rho_{73}(t), \rho_{68}(t)$ and $\rho_{86}(t)$, we also know that if only one of these four matrix element is not zero these two atoms are entangled. In figure. 2 we give the plot of the time evolution of the entanglement with different value of $\Gamma$, we set $\gamma=1$. It is shown that when $t=0$, there is no entanglement the reason is that the atomic system is in the initially state $|e \mu\rangle$. When $t$ is increased, the evolution of entanglement of this system is initially increased to a maximal value and then undergo a disentanglement procession. This procession is corresponding to the the process of photon exchange, it is the jump of atom $A|e\rangle \rightarrow|g\rangle$ (atom $B|\mu\rangle \rightarrow|g\rangle$ ) that make the absorption atom $B|g\rangle \rightarrow|e\rangle$ (atom $A$ $|g\rangle \rightarrow|\mu\rangle$ ) take place. We also observe that there exist a threshold time $t_{c}$ (for $\Gamma=0.6$, it is about 7.3), when $t$ is above the threshold time, the entanglement of this atomic system are always be zero, the reason is that when $T_{\mathcal{C}}$ is arrived, the radiation and absorption 
process is over, and the atomic system will be in the final unentangled state $|g g\rangle$. one can note that the threshold temperature $T_{c}$ is obviously improved when the value of $\Gamma$ is increased.

\section{Steady state entanglement with incoherent pumping}

From above section, one can see that with time evolution, entanglement finally disappeared due to the spontaneous emission. In order to obtain steady entanglement, now we introduce a incoherent pumping to our system. From level $|g\rangle$ to $|e\rangle(\mu\rangle)$, incoherent pumping can be modeled as the inverse process to spontaneous emission $[17,18]$, which means the master equation Eq.(6) should be added a third part $L_{I I I} \rho$ besides $\left(L_{I}+L_{I I}\right) \rho$, with

$$
\begin{aligned}
L_{I I I} \rho=\Omega_{1}[ & \left.\left(2 \sigma_{e g}^{A} \rho \sigma_{g e}^{A}-\sigma_{g g}^{A} \rho-\rho \sigma_{g g}^{A}\right)+A \rightarrow B\right] \\
& +\Omega_{2}\left[\left(2 \sigma_{\mu g}^{A} \rho \sigma_{g \mu}^{A}-\sigma_{\mu \mu}^{A} \rho-\rho \sigma_{\mu \mu}^{A}\right)+A \rightarrow B\right] .
\end{aligned}
$$

We can deduce differential equations as

$$
\begin{aligned}
\dot{\rho}_{11}= & -4 \gamma_{1} \rho_{11}+2 \Omega_{1}\left(\rho_{77}+\rho_{33}\right) \\
\dot{\rho}_{22}= & -2\left(\gamma_{1}+\gamma_{2}\right) \rho_{22}+2 \Omega_{1} \rho_{88}+2 \Omega_{2} \rho_{33}, \\
\dot{\rho}_{44}=- & 2\left(\gamma_{1}+\gamma_{2}\right) \rho_{44}+2 \Omega_{1} \rho_{66}+2 \Omega_{2} \rho_{77} \\
\dot{\rho}_{55}=- & 4 \gamma_{2} \rho_{55}+2 \Omega_{2}\left(\rho_{66}+\rho_{88}\right) \\
\dot{\rho}_{33}=- & \Gamma_{1}\left(\rho_{73}+\rho_{37}\right)-2 s_{1} \rho_{33}+2 \gamma_{2} \rho_{22} \\
& \quad+2 \gamma_{1} \rho_{11}+2 \Omega_{1} \rho_{99}, \\
\dot{\rho}_{37}=- & 2 \gamma_{1} \rho_{37}-\Gamma_{1}\left(\rho_{77}+\rho_{33}\right) \\
& \quad-2 s_{1} \rho_{37}+2 \Gamma_{1} \rho_{11}, \\
\dot{\rho}_{77}=- & \Gamma_{1}\left(\rho_{37}+\rho_{73}\right)-2 s_{1} \rho_{77} \\
& \quad+2 \gamma_{1} \rho_{11}+2 \gamma_{2} \rho_{44}+2 \Omega_{1} \rho_{99} . \\
\dot{\rho}_{66}=- & \Gamma_{2}\left(\rho_{86}+\rho_{68}\right)-2 s_{2} \rho_{66}+2 \gamma_{1} \rho_{44} \\
& \quad+2 \gamma_{2} \rho_{55}+2 \Omega_{2} \rho_{99}, \\
\dot{\rho}_{68}=- & \Gamma_{2}\left(\rho_{88}+\rho_{66}\right)-2 s_{2} \rho_{68}+2 \Gamma_{1} \rho_{55}, \\
\dot{\rho}_{88}=- & \Gamma_{2}\left(\rho_{86}+\rho_{68}\right)-2 s_{2} \rho_{88}+2 \gamma_{1} \rho_{22} \\
& +2 \gamma_{2} \rho_{55}+2 \Omega_{2} \rho_{99} \\
\dot{\rho}_{99}= & 2 \gamma_{1}\left(\rho_{33}+\rho_{77}\right)+2 \gamma_{2}\left(\rho_{66}+\rho_{88}\right) \\
& +2 \Gamma_{1}\left(\rho_{37}+\rho_{73}\right)+2 \Gamma_{2}\left(\rho_{68}+\rho_{86}\right)-4\left(\Omega_{1}+\Omega_{2}\right) \rho_{99} .
\end{aligned}
$$


where $s_{\alpha}=\gamma_{\alpha}+\Omega_{1}+\Omega_{2}(\alpha=1,2)$. We interest in getting the steady entanglement. Letting left side equal to zero, we deduce the steady state solution as

$$
\begin{aligned}
& \rho_{33}=\rho_{77}=\frac{b \gamma_{1} \gamma_{2}\left(\gamma_{1}+\gamma_{2}\right)}{P}, \\
& \rho_{66}=\rho_{88}=\frac{a}{b} \rho_{33}, \\
& \rho_{22}=\rho_{44}=\frac{1}{\gamma_{1}+\gamma_{2}}\left(\Omega_{1} \rho_{88}+\Omega_{2} \rho_{77}\right), \\
& \rho_{37}=\rho_{73}=\frac{\Gamma_{1}}{s_{1}}\left(\rho_{11}-\rho_{33}\right), \\
& \rho_{68}=\rho_{86}=\frac{\Gamma_{2}}{s_{2}}\left(\rho_{55}-\rho_{66}\right), \\
& \rho_{11}=\frac{\Omega_{1}}{\gamma_{1}} \rho_{33}, \\
& \rho_{99}=2 R_{1} \rho_{33}+2 R_{2} \rho_{66}
\end{aligned}
$$

with

$$
\begin{aligned}
& R_{\alpha}=\frac{s_{\alpha} \gamma_{\alpha}^{2}+\Gamma_{\alpha}^{2}\left(\Omega_{\alpha}-\gamma_{\alpha}\right)}{2 s_{\alpha} \gamma_{\alpha}\left(\Omega_{1}+\Omega_{2}\right)}, \alpha=1,2, \\
& a=\frac{\Gamma_{1}^{2}\left(\Omega_{1}-\gamma_{1}\right)}{s_{1} \gamma_{1}}+\frac{\Omega_{2} \gamma_{1}}{\gamma_{1}+\gamma_{2}}+\gamma_{1}-2 R_{1} \Omega_{1}, \\
& b=\frac{\Omega_{1} \gamma_{2}}{\gamma_{1}+\gamma_{2}}+2 R_{2} \Omega_{1}, \\
& P=2 \gamma_{1} \gamma_{2}\left(\gamma_{1}+\gamma_{2}\right)\left[\left(R_{1}+1\right) b+\left(R_{2}+1\right) a\right] \\
& \quad+\left(\gamma_{1}+\gamma_{2}\right)\left[\left(b \gamma_{2} \Omega_{1}+a \gamma_{1} \Omega_{2}\right)+\gamma_{1} \gamma_{2}\left(2 b \Omega_{2}+2 a \Omega_{1}\right)\right],
\end{aligned}
$$

Now, the entanglement negativity still be used, and the negative eigenvalue meet the equation

$$
\left(\rho_{11}-\lambda\right)\left(\rho_{55}-\lambda\right)\left(\rho_{99}-\lambda\right)-\left|\rho_{37}\right|^{2}\left(\rho_{55}-\lambda\right)-\left|\rho_{68}\right|^{2}\left(\rho_{11}-\lambda\right)=0 .
$$

In Fig. 3, we give the plot of the steady state negativity as a function of incoherent pumping $\Omega$. The rate of incoherent pumping $\Omega$ affect the steady state entanglement directly. If without the incoherent pumping, we can not obtain steady state entanglement which can be understood by section II. However, it is not mean that the larger rate of incoherent pumping the larger entanglement. In stead of that, the $\Omega$ have a ideal value where the entanglement is the largest. The conclusion is reasonable. The incoherent can overcome the decay of the atoms (expressed in Eq.(8)), however the overlarge incoherent pumping rate will also refrain the interchange absorption which is the true reason resulting in entanglement. Fig. 3 also show that increasing the value of $\Gamma$, the steady state entanglement is improved. So, appropriate rate of incoherent pumping help to produce steady entanglement. 

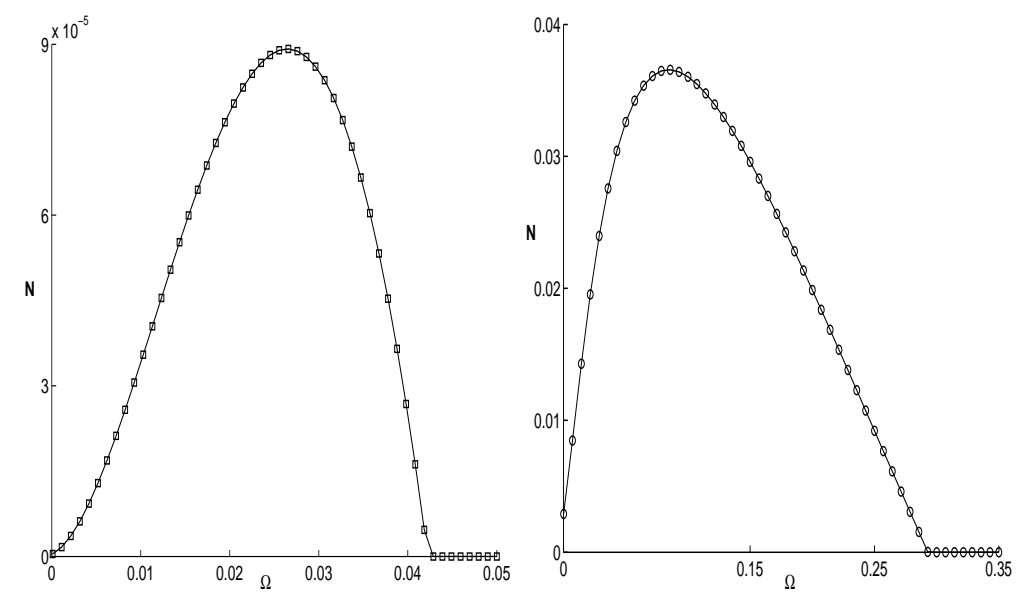

Figure 3: With $\gamma=1$, the steady negativity for our atomic system as a function of the incoherent pumping $\Omega$. For the square line (left figure): $\Gamma=0.5$, and the circle line (right figure): $\Gamma=0.9$.

\section{Conclusion}

In conclusion, it is analyzed the entanglement property in a system of two three-level atoms. We take into account the spontaneous emission and possible photon exchange between these two atoms when the distance is on the order of radiation wavelength. We find that this atomic system (initially in the existed states) could be entangled. There exist a threshold time $t_{c}$, when $t>t_{c}$ the entanglement are always be zero, but with $t<t_{c}$ it can be increased firstly and then decreased to zero. With increasing the value of $\Gamma$ can decrease the rate of disentanglement. Note that the time scale of evolution of entanglement is $t \sim \gamma^{-1}$. Hence in any typical dense multi-atom system, such entanglement is unavoidable. To obtain a steady state entanglement in such two- or multi-atom system, one can incoherently pump the excited states. We have shown that an appropriate rates of incoherent pump can help to produce entanglement.

\section{References}

[1] M. B. Plenio and S. F.Huelga, Phys. Rev. Lett. 88 (2002) 197901.

[2] M. B. Plenio, S. F. Huelga, A. Beige and P. L. Knight, Phys. Rev. A 59 (1998) 2468.

[3] K. H. Wu, Q. F. Huang, X. Q. Zhang, Advanced Materials Research, 662 (2013) 537.

[4] B. Duplantier, J. M. Raimond, V. Rivasseau (Eds.), Quantum Decoherence, Progress in Mathematical Physics, Vol. 48, (Springer Verlag, 2007).

[5] L. Derkacz and L. Jakobczyk, Phys. Rev. A 61 (2000) 062309.

[6] M. S. Kim, Jinhyoung Lee, D. Ahn and P. L. Knight, Phys. Rev. A 65 (2002) 040101.

[7] S. Schneider and G. J. Milburn, Phys. Rev. A 65 (2002) 042107.

[8] L. Jakobezyk, J. Phys. A: Math. Gen. 35 (2002) 6383. 
[9] G. S. Agarwal, Quantum Dynamical Semigroups and Applications (Lecture Notes in Phys. vol 286) (Berlin: Springer 1974).

[10] Z. Ficek, R. Tanas, Phys. Rep. 372 (2002) 369.

[11] T. Yu and J. H. Eberly, Quantum Inf Comput 7 (2007) 459.

[12] J. Evers, and C. H. Keitel, Phys. Rev. A 75 (2007) 032313.

[13] S. Hill and W. K. Wootters, Phys. Rev. Lett. 78 (1997) 5022.

[14] W. K. Wootters, Phys. Rev. Lett. 80 (1998) 2245.

[15] G. Vidal, R.F. Werner, Phys. Rev. A 65 (2002) 032314.

[16] K. Zyczkowski, P. Horodecki, A. Sanpera and M. Lewenstein, Phys. Rev. A 58 (1998) 883.

[17] Y. Mu and C. M. Savage, Phys. Rev. A 46 (1992) 5944.

[18] T. Salzburger and H. Ritsch, Phys. Rev. Leet. 93 (2004) 063002. 